\title{
Pectoralis Major and Pectoralis Minor Muscle Flap for Postpneumonectomy Empyema
}

\author{
Christo Dimitrov Shipkov ${ }^{1}$ and Angel Petrov Uchikov ${ }^{2}$ \\ ${ }^{1}$ Plastic and Craniofacial Ward, Department of Paediatric Surgery, and ${ }^{2}$ Department of Abdominal and Thoracic Surgery, Higher Medical \\ Institute - Plovdiv, Hirurgicheski kliniki, floor 3, \#66 Peshtersko Shosse Blvd, Plovdiv 4002, Bulgaria
}

The article by Nomori et al. ${ }^{1}$ has profoundly impressed us. Plastic and thoracic surgeons often collaborate because the vascularized muscle flaps are needed for the treatment of empyema. ${ }^{2}$

We have observed in our practice cases in which the latissimus dorsi muscle (LDM) was sectioned not only after posterolateral thoracotomy but after lateral thoracotomy as well. It is possible that the sectioning of the LDM not only at posterolateral thoracotomy might be much more frequent than is believed.

Since the thoracodorsal vascular pedicle and its hilus lies close to the lateral border of the LDM it can be easily sectioned when performing thoracotomy, the vascularization and, thus, the use of the LDM as a flap becoming uncertain. The importance of this fact was pointed out also by Malczewski et al. ${ }^{3}$

In treating empyema we have successfully used the LDM, even when sectioned, as a muscle flap on its lumbar and intercostal perforants for obliteration of the basal part of the empyema cavity. As mentioned by Nomori et al., ${ }^{1}$ the obliteration of the thoracic apex is quite difficult. In these cases the pectoral muscles are the closest source for vascularized tissue. Our anatomic studies showed that the vascular pedicle of the pectoral minor muscle (PMi) can be a branch either of the thoracoacromial artery, or a separate branch of the axillary artery or the lateral thoracic artery. In either case this branch lies very close to the primary pedicle of the pectoralis major muscle (the thoracoacromial artery), thus allowing the inclusion of the two muscles in one flap.
Based on this anatomical study, in January 2000 we used the pectoral major (PMa) and PMi muscles as a single flap in a case of postpneumonectomy empyema without bronchopleural fistula. The patient had undergone pneumonectomy for lung cancer. We performed intrathoracic transposition of the PMa and PMi muscles with simultaneous thoracoplasty. Excellent obliteration of the thoracic apex and the residual pleural cavity to the level of the third rib was achieved. The patient was dismissed on the 21st day without complications. He was free of empyema for 8 months when he presented with renal metastasis from his lung carcinoma.

We consider the PMa and PMi muscle flap proposed by Nomori et al. as excellent for obliteration of the thoracic apex in empyema patients, with minimal functional and aesthetic donor site deformity.

\section{References}

1. Nomori H, Horio H, Hasegawa T, Suemasu K. Intrathoracic transposition of a pectoralis major and pectoralis minor muscle flap for empyema in patients previously subjected to posterolateral thoracotomy. Surg Today 2001;31:295-9.

2. Nomori H, Horio H, Kobayashi R, Hasegawa T. Intrathoracic transposition of the musculocutaneous flap in treating empyema. Thorac Cardiovasc Surg 1995;43:171-5.

3. Malczewski MC, Colony L, Cobb LM. Latissimus-sparing thoracotomy in the pediatric patient: a valuable asset for thoracic reconstruction. J Pediatr Surg 1994;29;396-8. 